\title{
Evaluations of Hecke algebra traces at Kazhdan-Lusztig basis elements
}

\author{
Sam Clearman, Matthew Hyatt, Brittany Shelton, and Mark Skandera
}

Dept. of Mathematics, Lehigh University, 14 East Packer Ave., Bethlehem, PA 18015

\begin{abstract}
For irreducible characters $\left\{\chi_{q}^{\lambda} \mid \lambda \vdash n\right\}$ and induced sign characters $\left\{\epsilon_{q}^{\lambda} \mid \lambda \vdash n\right\}$ of the Hecke algebra $H_{n}(q)$, and Kazhdan-Lusztig basis elements $C_{w}^{\prime}(q)$ with $w$ avoiding the pattern 312 , we combinatorially interpret the polynomials $\chi_{q}^{\lambda}\left(q^{\frac{\ell(w)}{2}} C_{w}^{\prime}(q)\right)$ and $\epsilon_{q}^{\lambda}\left(q^{\frac{\ell(w)}{2}} C_{w}^{\prime}(q)\right)$. This gives a new algebraic interpretation of $q$-chromatic symmetric functions of Shareshian and Wachs. We conjecture similar interpretations and generating functions corresponding to other $H_{n}(q)$-traces.

Résumé. Pour les caractères irreductibles $\left\{\chi_{q}^{\lambda} \mid \lambda \vdash n\right\}$ et les caractères induits du signe $\left\{\epsilon_{q}^{\lambda} \mid \lambda \vdash n\right\}$ du algèbre de Hecke, et les éléments $C_{w}^{\prime}(q)$ du base Kazhdan-Lusztig avec $w$ qui évite le motif 312, nous interprétons les polinômes $\chi_{q}^{\lambda}\left(q^{\frac{\ell(w)}{2}} C_{w}^{\prime}(q)\right)$ et $\epsilon_{q}^{\lambda}\left(q^{\frac{\ell(w)}{2}} C_{w}^{\prime}(q)\right)$ de manière combinatorielle. Cette donne une nouvelle interprétation aux fonctionnes symétriques $q$-chromatiques de Shareshian et Wachs. Nous conjecturons des interprétations semblables et des foncionnes generatrices qui correspondent aux autres applications centrales de $H_{n}(q)$.
\end{abstract}

Keywords: Hecke algebra, trace, Kazhdan-Lusztig basis, tableau

\section{Introduction}

The symmetric group algebra $\mathbb{C S}_{n}$ and the (Iwahori-) Hecke algebra $H_{n}(q)$ have similar presentations as algebras over $\mathbb{C}$ and $\mathbb{C}\left[q^{\frac{1}{2}}, q^{-\frac{1}{2}}\right]$ respectively, with multiplicative identity elements $e$ and $T_{e}$, generators $s_{1}, \ldots, s_{n-1}$ and $T_{s_{1}}, \ldots, T_{s_{n-1}}$, and relations

$$
\begin{aligned}
& s_{i}^{2}=e \quad T_{s_{i}}^{2}=(q-1) T_{s_{i}}+q T_{e} \quad \text { for } i=1, \ldots, n-1, \\
& s_{i} s_{j} s_{i}=s_{j} s_{i} s_{j} \quad T_{s_{i}} T_{s_{j}} T_{s_{i}}=T_{s_{j}} T_{s_{i}} T_{s_{j}} \quad \text { for }|i-j|=1 \text {, } \\
& s_{i} s_{j}=s_{j} s_{i} \quad T_{s_{i}} T_{s_{j}}=T_{s_{j}} T_{s_{i}} \quad \text { for }|i-j| \geq 2 .
\end{aligned}
$$

Analogous to the natural basis $\left\{w \mid w \in \mathfrak{S}_{n}\right\}$ of $\mathbb{C S}_{n}$ is the natural basis $\left\{T_{w} \mid w \in \mathfrak{S}_{n}\right\}$ of $H_{n}(q)$, where we define $T_{w}=T_{s_{i_{1}}} \cdots T_{s_{i_{\ell}}}$ whenever $s_{i_{1}} \cdots s_{i_{\ell}}$ is a reduced expression for $w$ in $\mathfrak{S}_{n}$. We call $\ell$ the length of $w$ and write $\ell=\ell(w)$. (See [Hum90].) The specialization of $H_{n}(q)$ at $q^{\frac{1}{2}}=1$ is isomorphic to $\mathbb{C S}_{n}$. In addition to the natural bases of $\mathbb{C S}_{n}$ and $H_{n}(q)$, we have the (signless) KazhdanLusztig bases [KL79] $\left\{C_{w}^{\prime}(1) \mid w \in \mathfrak{S}_{n}\right\},\left\{C_{w}^{\prime}(q) \mid w \in \mathfrak{S}_{n}\right\}$, defined in terms of certain Kazhdan-Lusztig 1365-8050 @ 2013 Discrete Mathematics and Theoretical Computer Science (DMTCS), Nancy, France 
polynomials $\left\{P_{u, v}(q) \mid u, v \in \mathfrak{S}_{n}\right\}$ in $\mathbb{N}[q]$ by

$$
C_{w}^{\prime}(1)=\sum_{v \leq w} P_{v, w}(1) v, \quad C_{w}^{\prime}(q)=q_{e, w}^{-1} \sum_{v \leq w} P_{v, w}(q) T_{v},
$$

where $\leq$ denotes the Bruhat order and we define $q_{v, w}=q^{\frac{\ell(w)-\ell(v)}{2}}$. (See, e.g., [BB96].)

Representations of $\mathbb{C S}_{n}$ and $H_{n}(q)$ are often studied in terms of characters. The $\mathbb{C}$-span of the $\mathfrak{S}_{n^{-}}$ characters is called the space of $\mathfrak{S}_{n}$-class functions, and has dimension is equal to the number of integer partitions of $n$. (See [Sag01].) Three well-studied bases are the irreducible characters $\left\{\chi^{\lambda} \mid \lambda \vdash n\right\}$, induced sign characters $\left\{\epsilon^{\lambda} \mid \lambda \vdash n\right\}$, and induced trivial characters $\left\{\eta^{\lambda} \mid \lambda \vdash n\right\}$, where $\lambda \vdash n$ denotes that $\lambda$ is a partition of $n$. The $\mathbb{C}\left[q^{\frac{1}{2}}, q^{-\frac{1}{2}}\right]$-span of the $H_{n}(q)$-characters, called the space of $H_{n}(q)$-traces, has the same dimension and analogous character bases $\left\{\chi_{q}^{\lambda} \mid \lambda \vdash n\right\},\left\{\epsilon_{q}^{\lambda} \mid \lambda \vdash n\right\},\left\{\eta_{q}^{\lambda} \mid \lambda \vdash n\right\}$, specializing at $q^{\frac{1}{2}}=1$ to the $\mathfrak{S}_{n}$-character bases. Each of the two spaces has a fourth basis consisting of monomial class functions $\left\{\phi^{\lambda} \mid \lambda \vdash n\right\}$ or traces $\left\{\phi_{q}^{\lambda} \mid \lambda \vdash n\right\}$, and a fifth basis consisting of power sum class functions $\left\{\psi^{\lambda} \mid \lambda \vdash n\right\}$ or traces $\left\{\psi_{q}^{\lambda} \mid \lambda \vdash n\right\}$. These are defined via the inverse Kostka numbers $\left\{K_{\lambda, \mu}^{-1} \mid \lambda, \mu \vdash n\right\}$ and the numbers $\left\{L_{\lambda, \mu}^{-1} \mid \lambda, \mu \vdash n\right\}$ of row-constant Young tableaux of shape $\lambda$ and content $\mu$ by

$$
\phi^{\lambda} \underset{\operatorname{def}}{=} \sum_{\mu} K_{\lambda, \mu}^{-1} \chi^{\mu}, \quad \phi_{q}^{\lambda} \underset{\text { def }}{=} \sum_{\mu} K_{\lambda, \mu}^{-1} \chi_{q}^{\mu}, \quad \psi^{\lambda} \underset{\text { def }}{=} \sum_{\mu} L_{\lambda, \mu} \phi^{\mu}, \quad \psi_{q}^{\lambda} \underset{\text { def }}{=} \sum_{\mu} L_{\lambda, \mu} \phi_{q}^{\mu} .
$$

These functions are not characters. (See [BRW96], [Hai93], [Ste92].) In each space, the five bases are related to one another by the same transition matrices which relate the Schur, elementary, complete homogeneous, monomial, and power sum bases of the homogeneous degree $n$ symmetric functions. (See, e.g., [Sta99].)

It is known that irreducible $\mathfrak{S}_{n}$-characters $\left\{\chi^{\lambda} \mid \lambda \vdash n\right\}$ satisfy $\chi^{\lambda}(w) \in \mathbb{Z}$ for all $w \in \mathfrak{S}_{n}$. Thus for any integer linear combination $\theta$ of these and any element $z \in \mathbb{Z} \mathfrak{S}_{n}$, we have $\theta(z) \in \mathbb{Z}$ as well. In some cases, we may associate sets $R, S$ to the pair $(\theta, z)$ to combinatorially interpret the integer $\theta(z)$ as $(-1)^{|S|}|R|$. We summarize known results and open problems in the following table.

\begin{tabular}{|c|c|c|c|c|}
\hline$\theta$ & $\theta(w) \in \mathbb{N} ?$ & $\begin{array}{c}\text { interpretation of } \\
\theta(w) \text { as }(-1)^{|S|}|R| ?\end{array}$ & $\theta\left(C_{w}^{\prime}(1)\right) \in \mathbb{N} ?$ & $\begin{array}{c}\text { interpretation of } \\
\theta\left(C_{w}^{\prime}(1)\right) \text { as }|R| \text { for } \\
w \text { avoiding } 312 ?\end{array}$ \\
\hline$\eta^{\lambda}$ & yes & yes & yes & yes \\
$\epsilon^{\lambda}$ & no & yes & yes & yes \\
$\chi^{\lambda}$ & no & open & yes & yes \\
$\psi^{\lambda}$ & yes & yes & yes & yes \\
$\phi^{\lambda}$ & no & yes & conj. by Stembridge, Haiman & open \\
\hline
\end{tabular}

For known combinatorial interpretations of $\theta(w)$, see [BRW96]. The number $\chi^{\lambda}(w)$ may be computed by the well-known algorithm of Murnaghan and Nakayama. (See, e.g., [Sta99].) Otherwise, $\chi^{\lambda}(w)$ has no conjectured expression of the type stated above. Interpretations of $\theta\left(C_{w}^{\prime}(1)\right)$ are not known for general $w \in \mathfrak{S}_{n}$, but nonnegativity follows from work of Haiman [Hai93] and Stembridge [Ste91]. Interpretations of $\eta^{\lambda}\left(C_{w}^{\prime}(1)\right), \epsilon^{\lambda}\left(C_{w}^{\prime}(1)\right), \chi^{\lambda}\left(C_{w}^{\prime}(1)\right)$ for $w$ avoiding the pattern 312 follow via straightforward arguments from results of various authors, notably Gasharov [Gas96], Karlin-MacGregor [KM59], Lindström [Lin73], Littlewood [Lit40], Merris-Watkins [MW85], Stanley-Stembridge [SS93], [Ste91]. These 
will be discussed in Section 3. There is no conjectured combinatorial interpretation of $\phi^{\lambda}\left(C_{w}^{\prime}(1)\right)$, even for $w$ avoiding the pattern 312 although interpretations have been given for particular partitions $\lambda$ by Stembridge [Ste92] and several of the authors [CSS11].

It is known that irreducible $H_{n}(q)$-characters $\left\{\chi_{q}^{\lambda} \mid \lambda \vdash n\right\}$ satisfy $\chi_{q}^{\lambda}\left(T_{w}\right) \in \mathbb{Z}[q]$ for all $w \in \mathfrak{S}_{n}$. Thus for any integer linear combination $\theta_{q}$ of these and any element $z \in \operatorname{span}_{\mathbb{Z}[q]}\left\{T_{w} \mid w \in \mathfrak{S}_{n}\right\}$, we have $\theta_{q}(z) \in \mathbb{Z}[q]$ as well. In some cases, we may associate sequences $\left(S_{k}\right)_{k \geq 0},\left(R_{k}\right)_{\geq 0}$ of sets to the pair $\left(\theta_{q}, z\right)$ to combinatorially interpret $\theta_{q}(z)$ as $\sum_{k}(-1)^{\left|S_{k}\right|}\left|R_{k}\right| q^{k}$. We summarize known results and open problems in the following table.

\begin{tabular}{|c|c|c|c|c|}
\hline$\theta_{q}$ & $\theta_{q}\left(T_{w}\right) \in \mathbb{N}[q] ?$ & $\begin{array}{c}\text { interpretation of } \\
\theta_{q}\left(T_{w}\right) \text { as } \\
\sum_{k}(-1)^{\left|S_{k}\right|}\left|R_{k}\right| q^{k} ?\end{array}$ & $\theta\left(q_{e, w} C_{w}^{\prime}(q)\right) \in \mathbb{N}[q] ?$ & $\begin{array}{c}\text { interpretation of } \\
\theta_{q}\left(q_{e, w} C_{w}^{\prime}(q)\right) \text { as } \\
\sum_{k}\left|R_{k}\right| q^{k} \text { for } \\
w \text { avoiding 312? }\end{array}$ \\
\hline$\eta_{q}^{\lambda}$ & no & open & yes & conj. in Section 4 \\
$\epsilon_{q}^{\lambda}$ & no & open & yes & stated in Section 4 \\
$\chi_{q}^{\lambda}$ & no & open & yes & stated in Section 4 \\
$\psi_{q}^{\lambda}$ & no & open & conj. by Haiman & conj. in Section 4 \\
$\phi_{q}^{\lambda}$ & no & open & conj. by Haiman & open \\
\hline
\end{tabular}

The polynomial $\chi_{q}^{\lambda}\left(T_{w}\right)$, and therefore all polynomials $\theta_{q}\left(T_{w}\right)$, may be computed via a $q$-extension of the Murnaghan-Nakayama algorithm. (See, e.g., [KV84], [KW92], [Ram91].) Otherwise, $\theta_{q}^{\lambda}(w)$ has no conjectured expression of the type stated above. Interpretations of $\theta_{q}\left(q_{e, w} C_{w}^{\prime}(q)\right)$ are not known for general $w \in \mathfrak{S}_{n}$, but results concerning containment in $\mathbb{N}[q]$ follow principally from work of Haiman [Hai93]. For $w$ avoiding the pattern 312, a formula for $\epsilon_{q}^{\lambda}\left(q_{e, w} C_{w}^{\prime}(q)\right)$ is given by the authors in Section 4 . Work of Gasharov [Gas96] and Shareshian-Wachs [SW12] then implies a formula for $\chi_{q}^{\lambda}\left(q_{e, w} C_{w}^{\prime}(q)\right)$. Conjectures for $\psi_{q}^{\lambda}\left(q_{e, w} C_{w}^{\prime}(q)\right)$ are due to the authors and Shareshian-Wachs. These results and conjectures will also be discussed in Section 4 There is no conjectured combinatorial interpretation of $\phi_{q}^{\lambda}\left(q_{e, w} C_{w}^{\prime}(q)\right)$, even for $w$ avoiding the pattern 312 .

Another way to understand the evaluations $\theta(w)$ is to define a generating function $\operatorname{Imm}_{\theta}(x)$ in the polynomial ring $\mathbb{C}\left[x_{1,1}, \ldots, x_{n, n}\right]$ for $\left\{\theta(w) \mid w \in \mathfrak{S}_{n}\right\}$. Similarly, we may define a generating function $\operatorname{Imm}_{\theta_{q}}(x)$ in a certain noncommutative ring $\mathcal{A}(n ; q)$ for $\left\{\theta\left(T_{w}\right) \mid w \in \mathfrak{S}_{n}\right\}$. In some cases these generating functions have simple forms. We summarize known results in the following tables.

\begin{tabular}{|c|c|}
\hline$\theta$ & nice expression for $\operatorname{Imm}_{\theta}(x) ?$ \\
\hline$\eta^{\lambda}$ & yes \\
$\epsilon^{\lambda}$ & yes \\
$\chi^{\lambda}$ & open \\
$\psi^{\lambda}$ & yes \\
$\phi^{\lambda}$ & open \\
\hline
\end{tabular}

\begin{tabular}{|c|c|}
\hline$\theta_{q}$ & nice expression for $\operatorname{Imm}_{\theta_{q}}(x) ?$ \\
\hline$\eta_{q}^{\lambda}$ & yes \\
$\epsilon_{q}^{\lambda}$ & yes \\
$\chi_{q}^{\lambda}$ & open \\
$\psi_{q}^{\lambda}$ & conj. in Section 2 \\
$\phi_{q}^{\lambda}$ & open \\
\hline
\end{tabular}

Nice expressions for $\operatorname{Imm}_{\eta^{\lambda}}(x)$ and $\operatorname{Imm}_{\epsilon^{\lambda}}(x)$ are due to Littlewood [Lit40] and Merris-Watkins [MW85], and a nice expression for $\operatorname{Imm}_{\psi^{\lambda}}(x)$ follows immediately from the usual definition of $\psi$. An expression 
for $\operatorname{Imm}_{\chi^{\lambda}}(x)$ as a coefficient of a generating function in two sets of variables was given by GouldenJackson [GJ92]. There is no conjectured nice formula for $\operatorname{Imm}_{\phi^{\lambda}}(x)$, although a nice formula for particular partitions $\lambda$ was stated by Stembridge [Ste92]. These results will be discussed in Section 2. Nice expressions for $\operatorname{Imm}_{\eta_{q}^{\lambda}}(x)$ and $\operatorname{Imm}_{\epsilon_{q}^{\lambda}}(x)$ are due to the fourth author and Konvalinka [KS11], as is an expression for $\operatorname{Imm}_{\chi_{q}^{\lambda}}(x)$ as a coefficient in a generating function in two sets of variables. A nice expression for $\operatorname{Imm}_{\psi_{q}^{\lambda}}(x)$ is conjectured by the authors. These results and conjecture will be discussed in Section 2

In Section 2 we discuss known descriptions of the class functions in terms of generating functions in the ring $\mathbb{C}\left[x_{1,1}, \ldots, x_{n, n}\right]$ and in a certain quantum analog $\mathcal{A}(n ; q)$ of $\mathbb{C}\left[x_{1,1}, \ldots, x_{n, n}\right]$ known as the quantum matrix bialgebra. We also give a combinatorial interpretation of the entries of the transition matrices relating certain bases of $\mathcal{A}(n ; q)$. In Section 3 we give combinatorial interpretations, using results in the previous section. In Section 4 we give new descriptions of the class functions in terms of generating functions in the rings $\mathbb{C} \otimes \Lambda$ and $\mathbb{C}\left[q^{\frac{1}{2}}, q^{\frac{1}{2}}\right] \otimes \Lambda$ of symmetric functions having coefficients in $\mathbb{C}$ and $\mathbb{C}\left[q^{\frac{1}{2}}, q^{-\frac{1}{2}}\right]$. Finally, in Section 5 we draw connections to posets and to the chromatic quasisymmetric functions of Shareshian and Wachs.

\section{Generating functions for $\theta(w)$ and $\theta_{q}\left(T_{w}\right)$ when $\theta$ is fixed}

For a fixed $\mathfrak{S}_{n}$-class function $\theta$, we create a generating function for $\left\{\theta(w) \mid w \in \mathfrak{S}_{n}\right\}$ by writing $x=$ $\left(x_{i, j}\right), \mathbb{C}[x] \underset{\mathrm{def}}{=} \mathbb{C}\left[x_{1,1}, \ldots, x_{n, n}\right]$, and defining

$$
\operatorname{Imm}_{\theta}(x) \underset{\operatorname{def}}{=} \sum_{w \in \mathfrak{S}_{n}} \theta(w) x_{1, w_{1}} \cdots x_{n, w_{n}} \in \mathbb{C}[x] .
$$

We call this polynomial the $\theta$-immanant. The sign character $\left(w \mapsto(-1)^{\ell(w)}\right)$ immanant and trivial character $(w \mapsto 1)$ immanant are the determinant and permanent. Nice formulas for the $\epsilon^{\lambda}$-immanants and $\eta^{\lambda}$-immanants employ determinants and permanents of submatrices of $x$,

$$
x_{I, J} \underset{\mathrm{def}}{=}\left(x_{i, j}\right)_{i \in I, j \in J}, \quad I, J \subset[n] \underset{\mathrm{def}}{=}\{1, \ldots, n\} .
$$

In particular, for $\lambda=\left(\lambda_{1}, \ldots, \lambda_{r}\right) \vdash n$ we have Littlewood-Merris-Watkins identities [Lit40], [MW85]

$$
\operatorname{Imm}_{\epsilon^{\lambda}}(x)=\sum_{\left(I_{1}, \ldots, I_{r}\right)} \operatorname{det}\left(x_{I_{1}, I_{1}}\right) \cdots \operatorname{det}\left(x_{I_{r}, I_{r}}\right), \quad \operatorname{Imm}_{\eta^{\lambda}}(x)=\sum_{\left(I_{1}, \ldots, I_{r}\right)} \operatorname{per}\left(x_{I_{1}, I_{1}}\right) \cdots \operatorname{per}\left(x_{I_{r}, I_{r}}\right),
$$

where the sums are over all sequences of pairwise disjoint subsets of $[n]$ satisfying $\left|I_{j}\right|=\lambda_{j}$. A formula for the $\psi^{\lambda}$-immanant relies upon a sum over all permutations of cycle type $\lambda$,

$$
\operatorname{Imm}_{\psi^{\lambda}}(x)=z_{\lambda} \sum_{\substack{w \\ \operatorname{cyc}(w)=\lambda}} x_{1, w_{1}} \cdots x_{n, w_{n}}
$$

where $z_{\lambda}$ is the product $1^{\alpha_{1}} 2^{\alpha_{2}} \cdots n^{\alpha_{n}} \alpha_{1} ! \cdots \alpha_{n}$ !, and $\lambda$ has $\alpha_{i}$ parts equal to $i$ for $i=1, \ldots, n$. No such nice formulas are known for the $\chi^{\lambda}$-immanants or $\phi^{\lambda}$-immanants in general, although we do have a formula [Ste92, Thm. 2.8] for $\operatorname{Imm}_{\phi^{\lambda}}(x)$ when $\lambda_{1}=\cdots=\lambda_{r}=k$,

$$
\operatorname{Imm}_{\phi^{k^{r}}}(x)=\sum_{\left(I_{1}, \ldots, I_{k}\right)} \operatorname{det}\left(x_{I_{1}, I_{2}}\right) \operatorname{det}\left(x_{I_{2}, I_{3}}\right) \cdots \operatorname{det}\left(x_{I_{k}, I_{1}}\right),
$$


where the sum is over all sequences of pairwise disjoint subsets of $[n]=[k r]$ satisfying $\left|I_{j}\right|=r$.

For a fixed $H_{n}(q)$-trace $\theta_{q}$, we create a generating function for $\left\{\theta_{q}\left(T_{w}\right) \mid w \in \mathfrak{S}_{n}\right\}$ as before, but interpreting polynomials in $x=\left(x_{i, j}\right)$ as elements of the quantum matrix bialgebra $\mathcal{A}(n ; q)$, the noncommutative $\mathbb{C}\left[q^{\frac{1}{2}}, q^{-\frac{1}{2}}\right]$-algebra generated by $n^{2}$ variables $x=\left(x_{1,1}, \ldots, x_{n, n}\right)$, subject to the relations

$$
\begin{aligned}
x_{i, \ell} x_{i, k} & =q^{\frac{1}{2}} x_{i, k} x_{i, \ell}, & x_{j, k} x_{i, \ell} & =x_{i, \ell} x_{j, k} \\
x_{j, k} x_{i, k} & =q^{\frac{1}{2}} x_{i, k} x_{j, k} & x_{j, \ell} x_{i, k} & =x_{i, k} x_{j, \ell}+\left(q^{\frac{1}{2}}-q^{-\frac{1}{2}}\right) x_{i, \ell} x_{j, k},
\end{aligned}
$$

for all indices $1 \leq i<j \leq n$ and $1 \leq k<\ell \leq n$. As a $\mathbb{C}\left[q^{\frac{1}{2}}, q^{-\frac{1}{2}}\right]$-module, $\mathcal{A}(n ; q)$ has a basis of monomials $x_{\ell_{1}, m_{1}} \cdots x_{\ell_{r}, m_{r}}$ in which index pairs appear in lexicographic order. The relations (4) allow one to express other monomials in terms of this natural basis.

As a generating function for $\left\{\theta_{q}\left(T_{w}\right) \mid w \in \mathfrak{S}_{n}\right\}$, we define

$$
\operatorname{Imm}_{\theta_{q}}(x) \underset{\operatorname{def}}{=} \sum_{w \in \mathfrak{S}_{n}} \theta_{q}\left(T_{w}\right) q_{e, w}^{-1} x_{1, w_{1}} \cdots x_{n, w_{n}}
$$

in $\mathcal{A}(n ; q)$, and call this the $\theta_{q}$-immanant. The $H_{n}(q)$ sign character $\left(T_{w} \mapsto(-1)^{\ell(w)}\right)$ immanant and trivial character $\left(T_{w} \mapsto q^{\ell(w)}\right)$ immanant are called the quantum determinant and quantum permanent,

$$
\operatorname{det}_{q}(x)=\sum_{w \in \mathfrak{S}_{n}}\left(-q^{\frac{-1}{2}}\right)^{\ell(w)} x_{1, w_{1}} \cdots x_{n, w_{n}}, \quad \operatorname{per}_{q}(x)=\sum_{w \in \mathfrak{S}_{n}}\left(q^{\frac{1}{2}}\right)^{\ell(w)} x_{1, w_{1}} \cdots x_{n, w_{n}} .
$$

Specializing $\mathcal{A}(n ; q)$, $\operatorname{det}_{q}(x)$, and $\operatorname{per}_{q}(x)$ at $q^{\frac{1}{2}}=1$, we obtain the commutative polynomial ring $\mathbb{C}[x]$ and the classical determinant $\operatorname{det}(x)$ and permanent $\operatorname{per}(x)$.

Nice formulas for the $\epsilon_{q}^{\lambda}$-immanants and $\eta_{q}^{\lambda}$-immanants employ quantum determinants and quantum permanents of submatrices of $x$. In particular, the fourth author and Konvalinka [KS11, Thm. 5.4] proved quantum analogs of the Littlewood-Merris-Watkins identities in (3),

$\operatorname{Imm}_{\epsilon_{q}^{\lambda}}(x)=\sum_{\left(I_{1}, \ldots, I_{r}\right)} \operatorname{det}_{q}\left(x_{I_{1}, I_{1}}\right) \cdots \operatorname{det}_{q}\left(x_{I_{r}, I_{r}}\right), \quad \operatorname{Imm}_{\eta_{q}^{\lambda}}(x)=\sum_{\left(I_{1}, \ldots, I_{r}\right)} \operatorname{per}_{q}\left(x_{I_{1}, I_{1}}\right) \cdots \operatorname{per}_{q}\left(x_{I_{r}, I_{r}}\right)$,

where the sums are as in (3).

To state a nice form for the $\psi_{q}^{\lambda}$-immanant, we introduce the following definitions. Given a sequence $c=\left(i_{1}, \ldots, i_{k}\right)$ of distinct elements of $[n]$ with $i_{1}=\min \left\{i_{1}, \ldots, i_{k}\right\}$, define the element $d_{\left(i_{1}, \ldots, i_{k}\right)}(x)$ of $\mathcal{A}(n ; q)$ to be the sum of all cyclic rearrangements of the monomial $x_{i_{1}, i_{2}} x_{i_{2}, i_{3}} \cdots x_{i_{k}, i_{1}}$, each weighted by $q^{j-(k+1) / 2}$, where $x_{i_{1}, i_{2}}$ appears in position $j$,

$d_{c}(x)=q^{\frac{-(k-1)}{2}} x_{i_{1}, i_{2}} x_{i_{2}, i_{3}} \cdots x_{i_{k}, i_{1}}+q^{\frac{-(k-3)}{2}} x_{i_{k}, i_{1}} x_{i_{1}, i_{2}} \cdots x_{i_{k-1}, i_{k}}+\cdots+q^{\frac{(k-1)}{2}} x_{i_{2}, i_{3}} \cdots x_{i_{k}, i_{1}} x_{i_{1}, i_{2}}$.

For $w \in \mathfrak{S}_{n}$ having cycle type $\lambda=\left(\lambda_{1}, \ldots, \lambda_{r}\right)$, define the polynomial $g_{w}(x)$ to be the sum, over all cycle decompositions $\left(c_{1}, \ldots, c_{r}\right)$ of $w$ with $\left|c_{j}\right|=\lambda_{j}$, of $d_{c_{1}}(x) \cdots d_{c_{r}}(x)$. For example, the permutation $w=(1,4,3)(2,7)(5,6)=(1,4,3)(5,6)(2,7)$ with its (exactly) two admissible cycle decompositions leads to the element $g_{w}(x)=$

$$
\begin{gathered}
\left(q^{-1} x_{1,4} x_{4,3} x_{3,1}+x_{3,1} x_{1,4} x_{4,3}+q x_{4,3} x_{3,1} x_{1,4}\right)\left(q^{-\frac{1}{2}} x_{2,7} x_{7,2}+q^{\frac{1}{2}} x_{7,2} x_{2,7}\right)\left(q^{-\frac{1}{2}} x_{5,6} x_{6,5}+q^{\frac{1}{2}} x_{6,5} x_{5,6}\right)+ \\
\left(q^{-1} x_{1,4} x_{4,3} x_{3,1}+x_{3,1} x_{1,4} x_{4,3}+q x_{4,3} x_{3,1} x_{1,4}\right)\left(q^{-\frac{1}{2}} x_{5,6} x_{6,5}+q^{\frac{1}{2}} x_{6,5} x_{5,6}\right)\left(q^{\frac{1}{2}} x_{2,7} x_{7,2}+q^{\frac{1}{2}} x_{7,2} x_{2,7}\right) .
\end{gathered}
$$


Conjecture 2.1 Fix $\lambda=\left(\lambda_{1}, \ldots, \lambda_{r}\right) \vdash n$. Then in $\mathcal{A}(n ; q)$ we have $\operatorname{Imm}_{\psi_{q}^{\lambda}}(x)=\sum_{\substack{w \\ \operatorname{cyc}(w)=\lambda}} g_{w}(x)$.

For example, when $n=5$ and $\lambda=(3,2)$, we represent each permutation having cycle type $(3,2)$ as a product of a 3 -cycle with least letter written first and a 2 -cycle with least letter written first, $(1,2,3)(4,5)$, $(1,4,2)(3,5), \ldots,(3,5,4)(1,2)$, and we have

$$
\begin{aligned}
\operatorname{Imm}_{\psi_{q}^{32}}(x)= & \left(q^{-1} x_{1,2} x_{2,3} x_{3,1}+x_{3,1} x_{1,2} x_{2,3}+q x_{2,3} x_{3,1} x_{1,2}\right)\left(q^{-\frac{1}{2}} x_{4,5} x_{5,4}+q^{\frac{1}{2}} x_{5,4} x_{4,5}\right) \\
& +\left(q^{-1} x_{1,4} x_{4,2} x_{2,1}+x_{2,1} x_{1,4} x_{4,2}+q x_{4,2} x_{2,1} x_{1,4}\right)\left(q^{-\frac{1}{2}} x_{3,5} x_{5,3}+q^{\frac{1}{2}} x_{5,3} x_{3,5}\right) \\
& +\cdots+\left(q^{-1} x_{3,5} x_{5,4} x_{4,3}+x_{4,3} x_{3,5} x_{5,4}+q x_{5,4} x_{4,3} x_{3,5}\right)\left(q^{-\frac{1}{2}} x_{1,2} x_{2,1}+q^{\frac{1}{2}} x_{2,1} x_{1,2}\right) .
\end{aligned}
$$

No such nice formulas are known for the $\chi_{q}^{\lambda}$ - or $\phi_{q}^{\lambda}$ - immanants.

To obtain values of $\epsilon_{q}^{\lambda}\left(T_{w}\right)$ and $\eta_{q}^{\lambda}\left(T_{w}\right)$ from [5, one must use the relations 4 , to expand in the natural basis of (the zero-weight space $\operatorname{span}\left\{x_{1, w_{1}} \cdots x_{n, w_{n}} \mid w \in \mathfrak{S}_{n}\right\}$ of) $\mathcal{A}(n ; q)$. For this purpose, it is helpful to combinatorially interpret the coefficients arising as entries in the transition matrix relating the bases $\mathcal{B}_{u}=\left\{x_{u_{1}, v_{1}} \cdots x_{u_{n}, v_{n}} \mid v \in \mathfrak{S}_{n}\right\}$ and the natural basis $\left\{x_{1, w_{1}} \cdots x_{n, w_{n}} \mid w \in \mathfrak{S}_{n}\right\}$. These were obtained by Lambright and the fourth author in [LS10]. To combinatorially interpret the evaluations $\left\{\epsilon_{q}^{\lambda}\left(q_{e, w} C_{w}^{\prime}(q)\right) \mid \lambda \vdash n\right\}$ when $w$ avoids the pattern 312 , we prove a stronger result.

Theorem 2.2 Fix $u, w \in \mathfrak{S}_{n}$ with $u \leq w$, and let $s_{i_{1}} \cdots s_{i_{\ell}}$ be the right-to-left lexicographically greatest reduced expression for $u$. Choose an index $k \leq \ell+1$ and define $u^{\prime}=s_{i_{k-1}} \cdots s_{i_{1}} u, w^{\prime}=s_{i_{k-1}} \cdots s_{i_{1}} w$. Then we have

$$
x_{u_{1}, w_{1}} \cdots x_{u_{n}, w_{n}}=\sum_{v \in \mathfrak{S}_{n}} t_{u, w^{\prime}, v}\left(q^{\frac{1}{2}}-q^{-\frac{1}{2}}\right) x_{u_{1}^{\prime}, v_{1}} \cdots x_{u_{n}^{\prime}, v_{n}},
$$

where $\left\{t_{u, w^{\prime}, v}(q) \mid v \in \mathfrak{S}_{n}\right\}$ are polynomials in $\mathbb{N}[q]$. Moreover, the coefficient of $q^{b}$ in $t_{u, w^{\prime}, v}(q)$ is equal to the number of sequences $\left(\pi^{(0)}, \ldots, \pi^{(k-1)}\right)$ of permutations satisfying

1. $\pi^{0}=w, \pi^{(k-1)}=v$,

2. $\pi^{(j)} \in\left\{s_{i_{j}} \pi^{(j-1)}, \pi^{(j-1)}\right\}$ for $j=1, \ldots, k-1$,

3. $\pi^{(j)}=s_{i_{j}} \pi^{(j-1)}$ if $s_{i_{j}} \pi^{(j-1)}>\pi^{(j-1)}$,

4. $\pi^{(j)}=\pi^{(j-1)}$ for exactly $b$ values of $j$.

Proof: Omitted.

We may think of each sequence $\left(\pi^{(0)}, \ldots, \pi^{(k-1)}\right)$ in the above proof as a $(k-1)$-step walk from $w$ to $v$ in the weak order on $\mathfrak{S}_{n}$. After visiting $\pi^{(j)} \in \mathfrak{S}_{n}$, we may either revisit this permutation or move to $s_{i_{j}} \pi^{(j)}$, with the latter option being mandatory if $s_{i_{j}}$ is a left ascent for $\pi^{(j)}$.

\section{Descending star networks and interpretations of class functions}

Call a directed planar graph $G$ a planar network of order $n$ if it is acyclic and may be embedded in a disc so that $2 n$ boundary vertices labeled clockwise as source $1, \ldots$, source $n$ (with indegrees of 0 ) and 
sink $n, \ldots, \operatorname{sink} 1$ (with outdegrees of 0 ). In figures, we will draw sources on the left and sinks on the right, implicitly labeled $1, \ldots, n$ from bottom to top. Given a planar network $G$, define the path matrix $B=B(G)=\left(b_{i, j}\right)$ of $G$ by

$$
b_{i, j}=\text { number of paths in } G \text { from source } i \text { to sink } j .
$$

It is known that the path matrix of any planar network is totally nonnegative (TNN), i.e., that every minor of this matrix is nonnegative. This fact is known as Lindström's Lemma.

Call a sequence $\pi=\left(\pi_{1}, \ldots, \pi_{n}\right)$ of source-to-sink paths in a planar network a bijective path family if for some $w \in \mathfrak{S}_{n}$ with one-line notation $w_{1} \cdots w_{n}$, each component path $\pi_{i}$ begins at source $i$ and terminates at sink $w_{i}$. We will say also that $\pi$ has type $w$. Call a planar network a bijective skeleton if it is a union of $n$ source-to-sink paths. Clearly a bijective path family can cover an entire planar network $G$ only if $G$ is a bijective skeleton. For $[a, b]$ a subinterval of $[n]$, let $G_{[a, b]}$ be the bijective skeleton consisting of $a-1$ horizontal edges, a "star" of $b-a+1$ edges from sources $a, \ldots, b$ to an intermediate vertex, and $b-a+1$ more edges from this vertex to sinks $a, \ldots, b$, and $n-b$ more horizontal edges. For $n=4$, there are seven such networks: $G_{[1,4]}, G_{[2,4]}, G_{[1,3]}, G_{[3,4]}, G_{[2,3]}, G_{[1,2]}, G_{[1,1]}=\cdots=G_{[4,4]}$, respectively,

$$
* x^{*} \times \bar{x} \times \bar{x}=
$$

Define $G_{I} \circ G_{J}$ to be the concatenation of planar networks $G_{I}$ and $G_{J}$, and consider a sequence $\left(\left[c_{1}, d_{1}\right], \ldots,\left[c_{r}, d_{r}\right]\right)$ of subintervals of $[n]$ satisfying $c_{1}>\cdots>c_{r}$ and $d_{1}>\cdots>d_{r}$, and the concatenation $G_{\left[c_{1}, d_{1}\right]} \circ \cdots \circ G_{\left[c_{r}, d_{r}\right]}$ of corresponding star networks. For $n=4$, these are

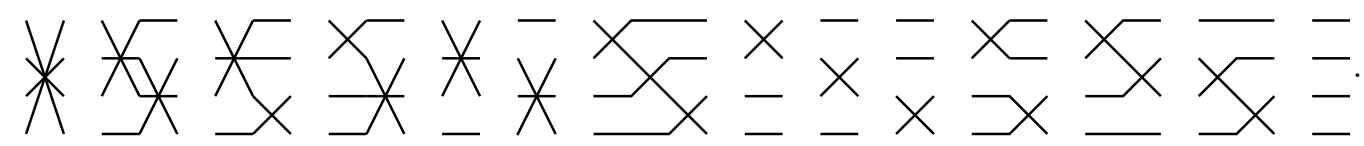

For each such planar network $G$, we define a related planar network $F$ by modifying $G$ as follows. For $i=1, \ldots, r-1$, if the intersection $\left[c_{i+1}, d_{i+1}\right] \cap\left[c_{i}, d_{i}\right]$ has cardinality $k>1$, then collapse the $k$ paths from the central vertex of $G_{\left[c_{i+1}, d_{i+1}\right]}$ to the central vertex of $G_{\left[c_{i}, d_{i}\right]}$, creating a single path between these vertices. Call $F$ a descending star network. For $n=4$, the decending star networks are

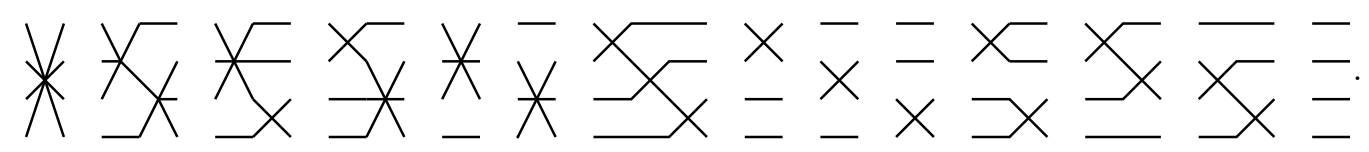

Proposition 3.1 There are $\frac{1}{n+1}\left(\begin{array}{c}2 n \\ n\end{array}\right)$ descending star networks of order $n$.

Proof: (Idea.) Let $F$ be the descending star network which corresponds as before $(7)$ to the concatenation $G=G_{\left[c_{1}, d_{1}\right]} \circ \cdots \circ G_{\left[c_{r}, d_{r}\right]}$. Modify $G$ to create the related network

$$
G^{\prime}=G_{\left[c_{1}, d_{1}\right]} \circ G_{\left[c_{1}, d_{1}\right] \cap\left[c_{2}, d_{2}\right]} \circ G_{\left[c_{2}, d_{2}\right]} \circ \cdots \circ G_{\left[c_{r-1}, d_{r-1}\right]} \circ G_{\left[c_{r-1}, d_{r-1}\right] \cap\left[c_{r}, d_{r}\right]} \circ G_{\left[c_{r}, d_{r}\right]}
$$

by inserting $G_{\left[c_{i}, d_{i}\right] \cap\left[c_{i+1}, d_{i+1}\right]}$ between $G_{\left[c_{i}, d_{i}\right]}$ and $G_{\left[c_{i+1}, d_{i+1}\right]}$ for $i=1, \ldots, r-1$. Now visually follow paths from sources to sinks, passing "straight" through each intersection, to complete a bijection to 312 avoiding permutations in $\mathfrak{S}_{n}$. For example, when $n=4$ and $F$ corresponds to $G=G_{[2,4]} \circ G_{[1,3]}$, we 
construct $G^{\prime}=G_{[2,4]} \circ G_{[2,3]} \circ G_{[1,3]}$ and obtain the 312-avoiding permutation $w=w(F)=3421$ :

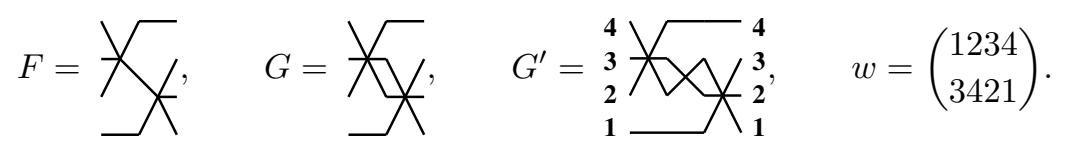

For each 312-avoiding permutation $w \in \mathfrak{S}_{n}$, let $F_{w}$ denote the descending star network corresponding to $w$ by the bijection in the proof of Proposition 3.1. Every descending star network $F_{w}$ is a bijective skeleton, and for every $v \leq w$ in the Bruhat order, there is exactly one bijective path family $\pi$ of type $v$ which covers $F_{w}$.

In a planar network $G$ of order $n$, the source-to-sink paths have a natural partial order $Q=Q(G)$. If $\pi_{i}$ is a path originating at source $i$, and $\rho_{j}$ is a path originating at source $j$, then we define $\pi_{i}<_{Q} \rho_{j}$ if $i<j$ and $\pi_{i}$ and $\rho_{j}$ never intersect. Observe that these conditions imply the index of the sink of $\pi_{i}$ to be less than the index of the sink of $\rho_{j}$. Let $P(G)$ be the subposet of $Q(G)$ induced by paths whose source and sink indices are equal. For each descending star network $F_{w}$, the poset $P\left(F_{w}\right)$ has exactly $n$ elements: there is exactly one path from source $i$ to sink $i$, for $i=1, \ldots, n$.

To combinatorially interpret evaluations of $\mathfrak{S}_{n}$-class functions and $H_{n}(q)$-traces, we will fill (French) Young diagrams with path families $\left(\pi_{1}, \ldots, \pi_{n}\right)$ covering a descending star network $F_{w}$, and will call the resulting structures $F$-tableaux. If an $F_{w}$-tableau $U$ contains a path family $\pi$ of type $v$, then we also say that $U$ has type $v$. We say that an $F_{w}$-tableau $U$ has shape $\lambda$ for some partition $\lambda=\left(\lambda_{1}, \ldots, \lambda_{r}\right)$ if it has $\lambda_{i}$ cells in row $i$ for all $i$. If $U$ has $\lambda_{i}$ cells in column $i$ for all $i$, we say that $U$ has shape $\lambda^{\top}$. In this case we define $\lambda^{\top}$ to be the partition whose $i$ th part is equal to the number of cells in row $i$ of $U$. Let $L(U)$ and $R(U)$ be the Young tableaux of integers obtained from $U$ by replacing paths $\pi_{1}, \ldots, \pi_{n}$ with their corresponding source and sink indices, respectively.

We define several properties of an $F$-tableau in terms of the poset $Q$ and the tableaux $L(U)$ and $R(U)$.

1. Call $U$ column-strict if whenever paths $\pi_{i_{1}}, \ldots, \pi_{i_{r}}$ appear from bottom to top in a column, then we have $\pi_{i_{1}}<_{Q} \cdots<_{Q} \pi_{i_{r}}$.

2. Call $U$ row-semistrict if whenever paths $\pi_{i_{1}}, \pi_{i_{2}}$ appear consecutively (from left to right) in a row, we have $\pi_{i_{1}}<_{Q} \pi_{i_{2}}$ or $\pi_{i_{1}}$ is incomparable to $\pi_{i_{2}}$ in $Q$.

3. Call $U$ cyclically row-semistrict if it is row-semistrict and the condition above applies also to paths $\pi_{i_{1}}, \pi_{i_{2}}$ appearing last and first (respectively) in the same row.

4. Call $U$ standard if it is column-strict and row-semistrict.

5. Call $U$ cylindrical if for each row of $L(U)$ containing indices $i_{1}, \ldots, i_{k}$ from left to right, the corresponding row of $R(U)$ contains $i_{2}, \ldots, i_{k}, i_{1}$ from left to right.

6. Call $U$ row-closed if $L(U)$ is row-strict (entries increase to the right) and if each row of $R(U)$ is a permutation of the corresponding row of $L(U)$.

For some $\mathfrak{S}_{n}$-class functions $\theta$, and all 312 -avoiding permutations $w$, we may combinatorially interpret $\theta\left(C_{w}^{\prime}(1)\right)$ in terms of a star network $F_{w}$ as follows.

Proposition 3.2 Let $w$ avoid the pattern 312, and let $F_{w}$ be the corresponding descending star network. 
1. $\eta^{\lambda}\left(C_{w}^{\prime}(1)\right)$ equals the number of row-semistrict $F_{w}$-tableaux of type e and shape $\lambda$. It also equals the number of row-closed $F_{w}$-tableaux of shape $\lambda$.

2. $\epsilon^{\lambda}\left(C_{w}^{\prime}(1)\right)$ equals the number of column-strict $F_{w}$-tableaux of type e and shape $\lambda^{\top}$.

3. $\chi^{\lambda}\left(C_{w}^{\prime}(1)\right)$ equals the number of semistandard $F_{w}$-tableaux of type e and shape $\lambda$.

4. $\psi^{\lambda}\left(C_{w}^{\prime}(1)\right)$ equals the number of cyclically row-semistrict $F_{w}$-tableaux of type e and shape $\lambda$. It also equals the number of cylindrical $F_{w}$-tableaux of shape $\lambda$.

5. For $\lambda_{1} \leq 2, \phi^{\lambda}\left(C_{w}^{\prime}(1)\right)$ equals zero if there exists a column-strict $F_{w}$-tableaux of type e and shape $\mu \prec \lambda$; otherwise it equals the number of column-strict $F_{w}$-tableaux of type e and shape $\lambda$.

6. For $\lambda=k^{r}, \phi^{\lambda}\left(C_{w}^{\prime}(1)\right)$ equals the number of column-strict cylindrical $F_{w}$-tableaux of shape $r^{k}$.

Proof: (Idea.) For $w$ avoiding 312, the path matrix $B=\left(b_{i, j}\right)$ of $F_{w}$ satisfies $\theta\left(C_{w}^{\prime}(1)\right)=\operatorname{Imm}_{\theta}(B)$.

Haiman [Hai93] and Stembridge [Ste91] have shown that we have $\chi^{\lambda}\left(C_{w}^{\prime}(1)\right) \geq 0$ for all $\lambda \vdash n$ and all $w \in \mathfrak{S}_{n}$. However, there is no conjectured combinatorial interpretation for $\chi^{\lambda}\left(C_{w}^{\prime}(1)\right)$ unless $w$ avoids 312. Haiman [Hai93] and Stembridge [Ste92] have also conjectured that we have $\phi^{\lambda}\left(C_{w}^{\prime}(1)\right) \geq 0$ for all $\lambda \vdash n$ and all $w \in \mathfrak{S}_{n}$. There is no general conjectured combinatorial interpretation for $\phi^{\lambda}\left(C_{w}^{\prime}(1)\right)$, even in the case that $w$ avoids 312, unless $\lambda$ has the special form stated in Proposition 3.2

\section{Statistics on $F$-tableaux and interpretations of $H_{n}(q)$-traces}

For $\theta$ an $\mathfrak{S}_{n}$-class function and $w$ avoiding 312, Proposition 3.2 interprets $\theta\left(C_{w}^{\prime}(1)\right)$ as the cardinality of a set of certain $F_{w}$-tableaux. For each of these sets of $F_{w}$-tableaux, we define a statistic mapping tableaux to nonnegative integers, and show (or conjecture) that $\theta_{q}\left(q_{e, w} C_{w}^{\prime}(q)\right)$ is a generating function for tableaux on which the statistic takes the values $k=0,1, \ldots$ In each case, our statistic is based upon the number of inversions of a permutation in $\mathfrak{S}_{n}$. Specifically, let $F$ be a descending star network, and let $U$ be an $F$-tableau containing path family $\pi=\left(\pi_{1}, \ldots, \pi_{n}\right)$ of type $w$. (Thus $\pi_{i}$ begins at source vertex $i$ and terminates at sink vertex $w_{i}$ for $i=1, \ldots, n$.) Let $\left(\pi_{i}, \pi_{j}\right)$ be a pair of intersecting paths in $F$ such that $\pi_{i}$ appears in a column of $U$ to the left of the column containing $\pi_{j}$. Call $\left(\pi_{i}, \pi_{j}\right)$ a left inversion in $U$ if we have $i>j$ and a right inversion in $U$ if we have $w_{i}>w_{j}$. Let $\operatorname{INV}(U)$ denote the number of left inversions in $U$, and let $\operatorname{RINV}(U)$ denote the number of right inversions in $U$.

Proofs of the validity of the tableaux interpretations in Proposition 3.2 depend upon a relationship between immanants and path matrices. To state a $q$-analog of this relationship, we define a map for each $n \times n$ complex matrix $B$ by

$$
\begin{aligned}
\sigma_{B}: \mathcal{A}(n ; q) & \rightarrow \mathbb{C}\left[q^{\frac{1}{2}}, q^{-\frac{1}{2}}\right] \\
x_{1, w_{1}} \cdots x_{n, w_{n}} & \mapsto q_{e, w} b_{1, w_{1}} \cdots b_{n, w_{n}}
\end{aligned}
$$

Proposition 4.1 Let $\theta_{q}$ be an $H_{n}(q)$-trace and let $w \in \mathfrak{S}_{n}$ avoid the pattern 312 . Then the path matrix $B$ of $F_{w}$ satisfies $\theta_{q}\left(q_{e, w} C_{w}^{\prime}(q)\right)=\sigma_{B}\left(\operatorname{Imm}_{\theta_{q}}(x)\right)$.

Proof: Omitted. 
Theorem 4.2 Let $w \in \mathfrak{S}_{n}$ avoid the pattern 312. For $\lambda \vdash n$ we have

$$
\epsilon_{q}^{\lambda}\left(q_{e, w} C_{w}^{\prime}(q)\right)=\sum q^{\mathrm{INV}(U)}
$$

where the sum is over all column-strict $F_{w}$-tableaux $U$ of type e and shape $\lambda^{\top}$. We also have

$$
\chi_{q}^{\lambda}\left(q_{e, w} C_{w}^{\prime}(q)\right)=\sum q^{\mathrm{INV}(U)}
$$

where the sum is over all standard $F_{w}$-tableaux $U$ of type e and shape $\lambda$.

Proof: Omitted. The proof of (10) depends upon a result of Shareshian and Wachs [SW12].

Let $U$ be an $F$-tableau of shape $\lambda=\left(\lambda_{1}, \ldots, \lambda_{r}\right)$ containing a path family $\pi$, and let $U_{i}$ be the $i$ th row of $U$. Let $U_{1} \circ \cdots \circ U_{r}$ and $U_{r} \circ \cdots \circ U_{1}$ be the $F$-tableaux of shape $n$ consisting of the rows of $U$ concatenated in increasing and decreasing order, respectively.

Conjecture 4.3 Let $w \in \mathfrak{S}_{n}$ avoid the pattern 312. For $\lambda \vdash n$ we have

$$
\eta_{q}^{\lambda}\left(q_{e, w} C_{w}^{\prime}(q)\right)=\sum q^{\mathrm{RINV}\left(U_{1} \circ \cdots \circ U_{r}\right)}
$$

where the sum is over all row-closed $F_{w}$-tableaux $U$ of shape $\lambda$. We also have

$$
\psi_{q}^{\lambda}\left(q_{e, w} C_{w}^{\prime}(q)\right)=\sum q^{\mathrm{INV}\left(U_{r} \circ \cdots \circ U_{1}\right)}
$$

where the sum is over all cylindrical $F_{w}$-tableaux $U$ of shape $\lambda$.

Haiman [Hai93] has shown that we have $\chi_{q}^{\lambda}\left(q_{e, w} C_{w}^{\prime}(q)\right) \in \mathbb{N}[q]$ for all $\lambda \vdash n$ and all $w \in \mathfrak{S}_{n}$. He has also conjectured that we have $\phi_{q}^{\lambda}\left(q_{e, w} C_{w}^{\prime}(q)\right) \in \mathbb{N}[q]$ for all $\lambda \vdash n$ and all $w \in \mathfrak{S}_{n}$. There is no general conjectured combinatorial interpretation for $\phi_{q}^{\lambda}\left(q_{e, w} C_{w}^{\prime}(q)\right)$, even in the case that $w$ avoids 312 .

\section{Generating functions for $\theta\left(C_{w}^{\prime}(1)\right), \theta_{q}\left(q_{e, w} C_{w}^{\prime}(q)\right)$ when $w$ is fixed}

For each $w \in \mathfrak{S}_{n}$, we define a symmetric generating function for values of $\theta\left(C_{w}^{\prime}(1)\right)$ by

$$
X_{w}=\sum_{\lambda \vdash n} \epsilon^{\lambda}\left(C_{w}^{\prime}(1)\right) m_{\lambda} \in \Lambda_{n} \underset{\operatorname{def}}{=} \operatorname{span}_{\mathbb{Z}}\left\{m_{\lambda} \mid \lambda \vdash n\right\} .
$$

Expanding $X_{w}$ in various bases of the space of homogeneous degree- $n$ symmetric functions, including the forgotten basis $\left\{f_{\lambda} \mid \lambda \vdash n\right\}$, we have

$$
X_{w}=\sum_{\lambda \vdash n} \eta^{\lambda}\left(C_{w}^{\prime}(1)\right) f_{\lambda}=\sum_{\lambda \vdash n} \chi^{\lambda^{\top}}\left(C_{w}^{\prime}(1)\right) s_{\lambda}=\sum_{\lambda \vdash n}(-1)^{n-\ell(\lambda)} \psi^{\lambda}\left(C_{w}^{\prime}(1)\right) \frac{p_{\lambda}}{z_{\lambda}}=\sum_{\lambda \vdash n} \phi^{\lambda}\left(C_{w}^{\prime}(1)\right) e_{\lambda},
$$

where $\ell(\lambda)$ is the number of (nonzero) parts of $\lambda$.

The function $X_{w}$ is related to the chromatic symmetric functions $\left\{X_{P} \mid P\right.$ a poset $\}$ of Stanley and Stembridge [Sta95], [SS93]: if $w$ avoids the pattern 312, then $X_{w}$ is equal to the Stanley-Stembridge chromatic symmetric function $X_{P\left(F_{w}\right)}$. On the other hand, not all chromatic symmetric functions $X_{P}$ can 
be expressed as $X_{w}$ for appropriate $w \in \mathfrak{S}_{n}$, nor can all generating functions $X_{w}$ be expressed as $X_{P}$ for an appropriate poset $P$. Stanley and Stembridge [Sta95], [SS93] have conjectured that $X_{P}$ is elementary nonnegative when $P$ has no induced subposet isomorphic to the disjoint union $(\mathbf{3}+\mathbf{1})$ of a three element chain and a single element. Call such a poset $(3+\mathbf{1})$-free. A special case of this conjecture is that $X_{w}$ is elementary nonnegative for $w$ avoiding 312. Haiman [Hai93] conjectured that $X_{w}$ is elementary nonnegative for all $w \in \mathfrak{S}_{n}$.

For each $w \in \mathfrak{S}_{n}$, we define a $\mathbb{Z}[q]$-symmetric generating function for values of $\theta_{q}\left(q_{e, w} C_{w}^{\prime}(q)\right)$ by

$$
X_{T_{w}}=\sum_{\lambda \vdash n} \epsilon_{q}^{\lambda}\left(q_{e, w} C_{w}^{\prime}(q)\right) m_{\lambda} \in \mathbb{Z}[q] \otimes \Lambda_{n}=\operatorname{span}_{\mathbb{Z}[q]}\left\{m_{\lambda} \mid \lambda \vdash n\right\} .
$$

Expanding $X_{T_{w}}$ in various bases of the homogeneous degree- $n$ graded component of $\mathbb{Z}[q] \otimes \Lambda_{n}$, we have $X_{T_{w}}=\sum_{\lambda \vdash n} \eta_{q}^{\lambda}\left(q_{e, w} C_{w}^{\prime}(q)\right) f_{\lambda}=\sum_{\lambda \vdash n} \chi_{q}^{\lambda^{\top}}\left(q_{e, w} C_{w}^{\prime}(q)\right) s_{\lambda}=\sum_{\lambda \vdash n} \frac{\psi_{q}^{\lambda}\left(q_{e, w} C_{w}^{\prime}(q)\right)}{(-1)^{n-\ell(\lambda)}} \frac{p_{\lambda}}{z_{\lambda}}=\sum_{\lambda \vdash n} \phi_{q}^{\lambda}\left(q_{e, w} C_{w}^{\prime}(q)\right) e_{\lambda}$.

The function $X_{T_{w}}$ specializes at $q=1$ to $X_{w}$, and is related to the chromatic quasisymmetric functions $\left\{X_{P, q} \mid P\right.$ a labeled poset $\}$ of Shareshian and Wachs [SW12], which specialize at $q=1$ to $X_{P}$. The function $X_{P, q}$ is itself symmetric (i.e., it belongs to $\left.\mathbb{Z}[q] \otimes \Lambda_{n}\right)$ when $P$ is $(\mathbf{3}+\mathbf{1})$-free, $(\mathbf{2}+\mathbf{2})$-free, and labeled strategically. If $w$ avoids the pattern 312 , then by Theorem 4.2, $X_{T_{w}}$ is equal to the ShareshianWachs chromatic symmetric function $X_{P\left(F_{w}\right), q}$, with each element of $P\left(F_{w}\right)$ labeled according to the source and sink of the path in $F_{w}$ it represents. Again, not all chromatic symmetric functions $X_{P, q}$ can be expressed as $X_{T_{w}}$ for appropriate $w \in \mathfrak{S}_{n}$, nor can all generating functions $X_{T_{w}}$ be expressed as $X_{P, q}$ for an appropriate labeled poset $P$. Shareshian and Wachs [SW12] conjectured that $X_{P, q}$ belongs to $\operatorname{span}_{\mathbb{N}[q]}\left\{e_{\lambda} \mid \lambda \vdash n\right\}$ when $P$ is $(\mathbf{3}+\mathbf{1})$-free, $(\mathbf{2}+\mathbf{2})$-free, and labeled appropriately. By Theorem 4.2 this is equivalent to the conjecture that $X_{T_{w}}$ belongs to $\operatorname{span}_{\mathbb{N}[q]}\left\{e_{\lambda} \mid \lambda \vdash n\right\}$ for $w$ avoiding 312 . Haiman [Hai93] conjectured that $X_{T_{w}}$ belongs to $\operatorname{span}_{\mathbb{N}[q]}\left\{e_{\lambda} \mid \lambda \vdash n\right\}$ for all $w \in \mathfrak{S}_{n}$.

\section{References}

[BB96] A. Björner and F. Brenti. An improved tableau criterion for Bruhat order. Electron. J. Combin., 3(1), 1996. Research paper 22, 5 pp. (electronic).

[BRW96] D. Beck, J. Remmel, and T. Whitehead. The combinatorics of transition matrices between the bases of the symmetric functions and the $b_{n}$ analogues. Discrete Math., 153:3-27, 1996.

[CSS11] Sam Clearman, Brittany Shelton, and Mark Skandera. Path tableaux and combinatorial interpretations of immanants for class functions on $S_{n}$. In 23rd International Conference on Formal Power Series and Algebraic Combinatorics (FPSAC 2011), Discrete Math. Theor. Comput. Sci. Proc., AO, pages 233-244. Assoc. Discrete Math. Theor. Comput. Sci., Nancy, 2011.

[Gas96] V. Gasharov. Incomparability graphs of $(\mathbf{3}+\mathbf{1})$-free posets are $s$-positive. Discrete Math., 157:211-215, 1996.

[GJ92] I. P. Goulden and D. M. Jackson. Immanants, Schur functions, and the MacMahon master theorem. Proc. Amer. Math. Soc., 115(3):605-612, 1992.

[Hai93] M. Haiman. Hecke algebra characters and immanant conjectures. J. Amer. Math. Soc., 6(3):569$595,1993$. 
[Hum90] James E. Humphreys. Reflection groups and Coxeter groups. Cambridge University Press, 1990.

[KL79] D. Kazhdan and G. Lusztig. Representations of Coxeter groups and Hecke algebras. Invent. Math., 53:165-184, 1979.

[KM59] S. Karlin and G. McGregor. Coincidence probabilities. Pacific J. Math., 9:1141-1164, 1959.

[KS11] Matjaž Konvalinka and Mark Skandera. Generating functions for Hecke algebra characters. Canad. J. Math., 63(2):413-435, 2011.

[KV84] S. V. Kerov and A. M. Vershik. Characters, factor representations and $K$-functor of the infinite symmetric group. In Operator algebras and group representations, Vol. II (Neptun, 1980), volume 18 of Monogr. Stud. Math., pages 23-32. Pitman, Boston, MA, 1984.

[KW92] R. C. King and B. G. Wybourne. Representations and traces of the Hecke algebras $H_{n}(q)$ of type $A_{n-1}$. J. Math. Phys., 33(1):4-14, 1992.

[Lin73] B. Lindström. On the vector representations of induced matroids. Bull. London Math. Soc., 5:85-90, 1973.

[Lit40] Dudley E. Littlewood. The Theory of Group Characters and Matrix Representations of Groups. Oxford University Press, New York, 1940.

[LS10] J. Lambright and M. Skandera. Combinatorial formulas for double parabolic $R$-polynomials. In Proceedings of the 22nd Interntational Conference on Formal Power Series and Algebraic Combinatorics (FPSAC 2010), Discrete Math. Theor. Comput. Sci. Proc., AO, pages 857-868. Assoc. Discrete Math. Theor. Comput. Sci., Nancy, 2010.

[MW85] Russell Merris and William Watkins. Inequalities and identities for generalized matrix functions. Linear Algebra Appl., 64:223-242, 1985.

[Ram91] Arun Ram. A Frobenius formula for the characters of the Hecke algebras. Invent. Math., 106(3):461-488, 1991.

[Sag01] B. Sagan. The Symmetric Group. Springer, New York, 2001.

[SS93] R. Stanley and J. R. Stembridge. On immanants of Jacobi-Trudi matrices and permutations with restricted positions. J. Combin. Theory Ser. A, 62:261-279, 1993.

[Sta95] R. Stanley. A symmetric function generalization of the chromatic polynomial of a graph. Adv. Math., 111:166-194, 1995.

[Sta99] R. Stanley. Enumerative Combinatorics, volume 2. Cambridge University Press, Cambridge, 1999.

[Ste91] John Stembridge. Immanants of totally positive matrices are nonnegative. Bull. London Math. Soc., 23:422-428, 1991.

[Ste92] John Stembridge. Some conjectures for immanants. Can. J. Math., 44(5):1079-1099, 1992.

[SW12] John Shareshian and Michelle Wachs. Chromatic quasisymmetric functions and Hessenberg varieties. In A Bjorner, F Cohen, C De Concini, C Procesi, and M Salvetti, editors, Configuration Spaces, pages 433-460, Pisa, 2012. Edizione Della Normale. 